\title{
Continuous Intravenous Morphine Infusion for Severe Dyspnea in Terminally Ill Interstitial Pneumonia Patients
}

\author{
Makiko Takeyasu ${ }^{1}$, Atsushi Miyamoto ${ }^{1}$, Daisuke Kato ${ }^{2}$, Yui Takahashi ${ }^{1}$, Kazumasa Ogawa ${ }^{1}$, \\ Kyoko Murase ${ }^{1}$, Sayaka Mochizuki ${ }^{1}$, Shigeo Hanada ${ }^{1}$, Hironori Uruga ${ }^{1}$, Hisashi Takaya ${ }^{1}$, \\ Nasa Morokawa ${ }^{1}$ and Kazuma Kishi ${ }^{1,3}$
}

\begin{abstract}
Objective The aims of this study were to evaluate the efficacy and safety of continuous morphine infusion for dyspnea in patients with acute exacerbation (AE) of end-stage interstitial pneumonia (IP).

Methods We conducted a retrospective study. Based on the subjective clinical effectiveness ratings of "good," "moderate," "poor," or "unknown," the efficacy of continuous morphine infusion treatment was evaluated as defined as symptom relief that was "good" or "moderate."

Patients This study included 22 consecutive opioid-naïve patients who received continuous morphine infusion in the palliative treatment of dyspnea resulting from AE-IP.

Results Of 22 patients, nine achieved good dyspnea relief, eight had moderate relief, four had a poor response and one response was "unknown" within 24 hours of starting morphine infusion. Using an operational definition of dyspnea relief that was rated "good" or "moderate," the efficacy rate of morphine was $77 \%$ (n= 17). There was a significant change in the respiratory rate $(25$ respirations per minute at baseline vs. 17 respirations per minute after 12 hours, $\mathrm{p}=0.02$ ), however, none of the patients studied had fewer than eight respirations per minute.

Conclusion We conclude that continuous morphine infusion is an effective and safe therapy for severe dyspnea in terminal AE-IP patients without any serious adverse events.
\end{abstract}

Key words: interstitial pneumonia, morphine, acute exacerbation, idiopathic pulmonary fibrosis, palliative care

(Intern Med 55: 725-729, 2016)

(DOI: 10.2169/internalmedicine.55.5362)

\section{Introduction}

Acute exacerbation (AE) of interstitial pneumonia (IP) is characterized by the development of an acute lung injury, typically resulting in acute respiratory distress, in a patient with a preexisting fibrosing IP, including idiopathic pulmonary fibrosis (IPF) and other types of chronic IP, such as IP associated with collagen vascular disease (1). Even in the absence of clear evidence of effectiveness, high-dose steroids are the usual primary treatment of AE-IP (1). The prognosis of patients with $\mathrm{AE}$ is very poor, with an average mortality of $70 \%$ (2). When treatment is not effective and the prognosis is considered to be only a few days, irreversible respiratory failure proceeds rapidly, resulting in severe dyspnea. To relieve the symptoms, oxygen supplements and antipsychotics are given empirically, often with unsatisfactory results. An appropriate method of palliative care is needed, and yet the evidence in this domain is lacking.

Morphine is known to be effective for the relief of dyspnea in patients with malignant disease (3) and is widely used for such patients. Recently, the effectiveness of morphine for dyspnea in patients with non-malignant respiratory disease, including chronic obstructive pulmonary disease

${ }^{1}$ Department of Respiratory Medicine, Respiratory Center, Toranomon Hospital, Japan, ${ }^{2}$ Department of Palliative Medicine, Toranomon Hospital, Japan and ${ }^{3}$ Okinaka Memorial Institute for Medical Research, Japan

Received for publication March 16, 2015; Accepted for publication June 24, 2015

Correspondence to Dr. Makiko Takeyasu, makikotakeyasu@gmail.com 
Table 1. Definitions Used in Measurement of Efficacy.

\begin{tabular}{|c|c|}
\hline Good & $\begin{array}{l}\text { Chart descriptions of patient expressions such as } \\
\text { "I don't feel dyspnea anymore," or "dyspnea is relieved" } \\
\text { or } \\
\text { Physician or nursing records with observations such as } \\
\text { "patient doesn't seem to feel dyspnea," and "dyspnea seems } \\
\text { to have been relieved" }\end{array}$ \\
\hline Moderate & $\begin{array}{l}\text { Chart descriptions of patient expressions such as, "dyspnea } \\
\text { is relieved, but I still feel it," and "I feel better than before" } \\
\text { or } \\
\text { Physician or nursing records with observations such as } \\
\text { "dyspnea seems to have been relieved better than before," } \\
\text { and "patient seems to be more calm than before" }\end{array}$ \\
\hline Poor & $\begin{array}{l}\text { Chart descriptions of patient expressions such as "I still feel } \\
\text { dyspnea," "dyspnea is not relieved" } \\
\text { or } \\
\text { Physician or nursing records with observations such as } \\
\text { "patient still seems to feel dyspnea" and "dyspnea does not } \\
\text { seem to have relieved" }\end{array}$ \\
\hline Unknown & Absence of description about change in symptom severity \\
\hline
\end{tabular}

(COPD), has been reported in several studies (4-6). A small prospective study by Allen et al. found that diamorphine was effective for dyspnea relief in terminal IPF patients (7). However, there is a paucity of high-quality studies evaluating the role of opioids in the management of dyspnea in IP patients. Furthermore, because the lack of progressive respiratory failure was one of the inclusion criteria in these studies, it is unclear whether morphine is effective and safe for patients with progressive respiratory failure and a prognosis of a few days. Therefore, we retrospectively studied the treatment of terminal IP patients with AE and evaluated the efficacy and safety of continuous intravenous morphine infusion for these patients.

\section{Materials and Methods}

We studied consecutive patients who died of AE-IP from April 2006 through March 2013. A diagnosis of AE was made following the criteria of IPF in the 2011 consensus guideline for the diagnosis and treatment of IP in Japan (8). We included both the confirmed cases of $\mathrm{AE}$ and the patients who might have both $\mathrm{AE}$ and infection, because the prognoses of those are substantially the same (9). We excluded patients with delirium (as diagnosed by the DSM-IV: Diagnostic and Statistical Manual of Mental Disorders, fourth edition), as the evaluation of the symptoms would be unreliable.

We conducted a retrospective study based on a chart review. Background data obtained included medications or other treatments for dyspnea. Patient characteristics, other medications administered for dyspnea, the duration of treatment from the initial administration to death, and the initial and maximum doses of morphine were also obtained for those patients who were treated with continuous morphine infusion. We evaluated the efficacy of morphine in these patients. Additionally, we evaluated temporal changes in the vital signs, including the respiratory rate and blood pressure, in 11 patients who had chart descriptions of vital signs, these data were evaluated before starting continuous morphine infusion treatment and 12 hours afterward.

Although a numerical rating scale is typically used to measure the severity of dyspnea, it is often difficult to measure severe dyspnea in end-stage patients with progressive respiratory failure. This is because their symptoms are so severe and progress so rapidly that patients can barely speak or evaluate their own symptoms. Not surprisingly, the evidence base to guide treatment decisions in palliative care units is lacking. Acknowledging this gap, a previous study in terminally ill patients with cancer sought to clarify the comparative efficacy between midazolam and flunitrazepam for primary insomnia in terminally ill patients with cancer, a setting in which the severity of the patients' illness necessitated the use of an ad hoc symptom rating scale (10). We similarly used an ad hoc scale in the present study due to the lack of a validated measurement tool for symptom evaluation in patients with severe dyspnea (Table 1) on the day of initial administration to measure the effectiveness of morphine infusion. We designed the evaluation methods to strictly follow the actual chart description to minimize bias due to a retrospective assessment. The effectiveness was rated as "good," "moderate," "poor," or "unknown." The efficacy rate was defined as the ratio of patients with "good" and "moderate" ratings compared with the total number of patients in the study.

Additionally evaluated were changes in the vital signs including: the respiratory rate, oxygen saturation, temporal changes in the systolic blood pressure from the initiation of continuous morphine infusion to 12 hours after starting continuous morphine infusion, and the presence or absence of adverse effects of morphine, including delirium, sedation, and respiratory or cardiac depression.

We used the Wilcoxon signed rank test to test for trends among continuous variables, including the vital signs. Twotailed $\mathrm{p}$ values were utilized and statistical significant was considered to exist at $\mathrm{p}<0.05$. We used descriptive statistics to summarize the population's characteristics, breathlessness, and adverse events. All data were analyzed using the SPSS software program (for Windows, version 11.0, SPSS Inc., Chicago, USA).

A signed consent form for the administration of opioids was obtained from each patient and/or his family as per our routine management of patients with AE-IP. The study protocol was approved by the Institutional Review Board of Toranomon Hospital (No.824).

\section{Results}

A total of 44 patients died due to AE-IP during the study period. Excluding 10 patients incapable of communication (4 patients were intubated and 6 patients were somnolent due to carbon dioxide narcosis or other reasons), all patients complained of dyspnea at rest. Among those 34 patients, 25 


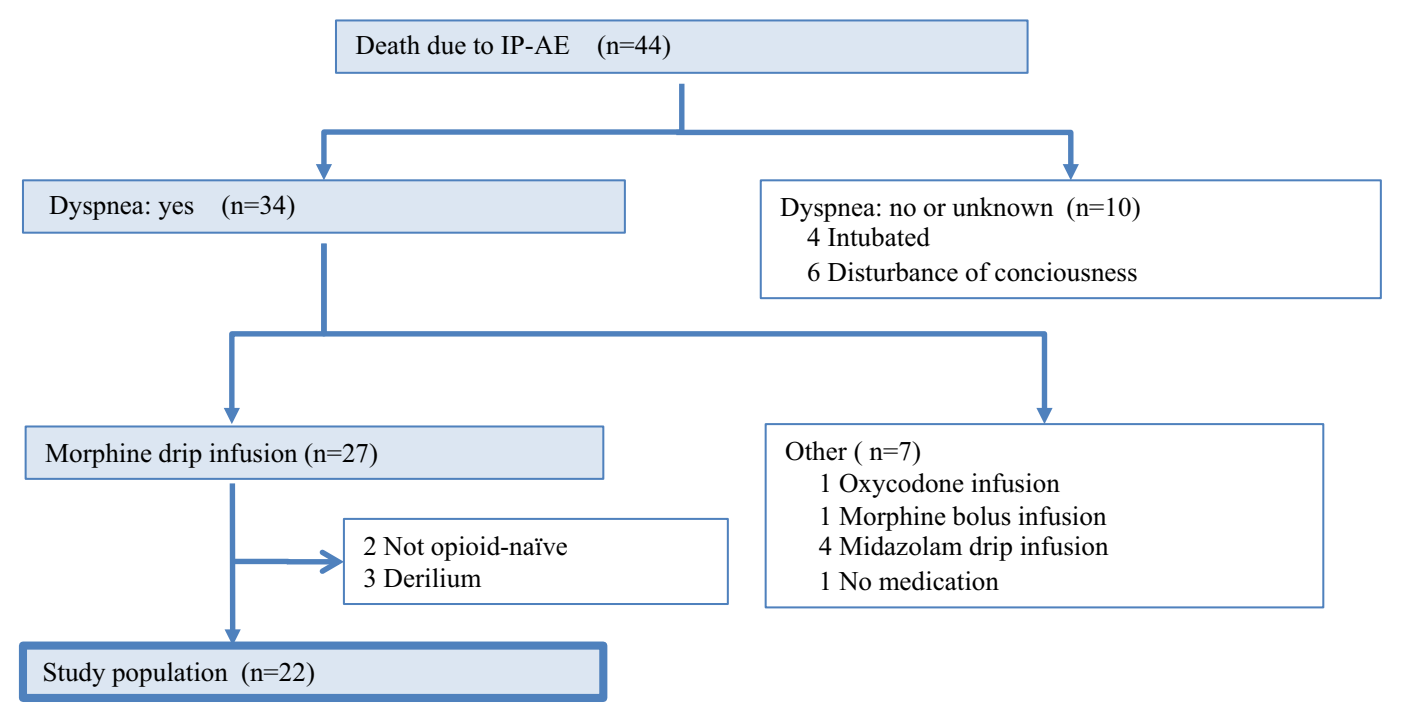

Figure 1. Study flowchart.

Table 2. Patient Characteristics of Study Population $(n=22)$.

\begin{tabular}{lr}
\hline & $\mathrm{n}(\%)$ \\
\hline Median age in years (range) & $72(59-86)$ \\
ECOG performance status at 4 & $22(100)$ \\
Male gender & $17(77)$ \\
Type of interstitial pneumonia (IP) & \\
$\quad$ Idiopathic pulmonary fibrosis (IPF) & $13(59)$ \\
$\quad$ Other & $9(41)$ \\
$\quad$ Nonspecific interstitial pneumonia (NSIP) & 1 \\
$\quad$ Collagen vascular disease associated Interstitial pneumonia & 3 \\
$\quad$ Unclassifiable & 5 \\
Current or past smoker & $19(86)$ \\
Treatment approach for acute exacerbation & \\
$\quad$ Steroid pulse therapy & $22(100)$ \\
Cyclosporine A & $12(55)$ \\
Cyclophosphamide pulse therapy & $1(5)$ \\
$\quad$ Noninvasive positive pressure ventilation & $7(32)$ \\
Receiving supplemental oxygen & $22(100)$ \\
$\quad>10$ L per minute & $21(95)$ \\
\hline ECOG : Eastern Cooperative Oncology Group &
\end{tabular}

ECOG : Eastern Cooperative Oncology Group

opioid-naïve patients received continuous intravenous morphine infusion. Among those 25 patients, 3 patients who had delirium before starting morphine were excluded from further analysis. Fig. 1 summarizes the study flowchart.

Table 2 summarizes the clinical features of 22 patients who received continuous morphine infusion and who did not have delirium before starting morphine. They included 17 men, 59 to 86 years of age (median age, 72 years). The median time from the initial diagnosis of IP was 2 years (range $0-14)$. The numbers of patients with IPF/UIP (usual interstitial pneumonia) and other types of IP were 13 and 9 patients, respectively. The number of patients who received oxygen supplement of $>10 \mathrm{~L}$ per minute was 21 .

The median respiratory rate and average systolic blood pressure and oxygen saturation, as measured by pulse oximetry at rest on supplemental oxygen, before starting morphine was 25 respirations per minute, $126 \mathrm{mmHg}$, and $89 \%$, respectively. Patients tended to be tachypneic and severely hypoxic.
As per the standard management of AE, all patients had received high-dose steroid therapy (methylprednisolone $1 \mathrm{~g}$, administered once daily for three days) for a median of three times (range 1-4), and seven had received noninvasive positive pressure ventilation for respiratory failure before starting morphine infusion.

Fig. 2 shows the morphine infusion protocol. Morphine was started when both the physicians and nurses thought that dyspnea was not relieved by any other methods, including oxygen or sedative agents, and that the prognosis of the patient was only a few days. Morphine was dissolved in normal saline, usually 50 or $100 \mathrm{~mL}$, and was started as continuous intravenous drip infusion. The median administration period of morphine infusion was two days (range 028 ). The median initial and maximum doses were 0.4 (range $0.2-0.8$ ) $\mathrm{mg}$ per hour and 0.8 (range 0.2-2.9) $\mathrm{mg}$ per hour. The initial dose in many cases was $0.2-0.4 \mathrm{mg} / \mathrm{h}$, equivalent to $5-10 \mathrm{mg} /$ day, which was decided by the physician in accordance with the official statement of the American Thoracic Society (11). Dose escalation by $0.2 \mathrm{mg} / \mathrm{h}$ was allowed after starting morphine if the symptom was not relieved, the physician thought that dose escalation could be effective, and that there were no signs of respiratory or cardiac depression. Five patients died within 24 hours. All causes of death were considered to be respiratory failure, because most of these patients had a severe respiratory failure status before starting morphine (patients were hypoxic, with oxygen saturation $<85 \%$ before starting morphine).

We evaluated the efficacy of morphine in these 22 patients, none with delirium before starting morphine. Nine patients achieved good dyspnea relief, eight had moderate relief, and four had a poor response (one response was characterized as "unknown") within 24 hours after starting morphine infusion. The efficacy rate of morphine was $77 \%$. As respiratory failure progressed, dyspnea worsened and $11 \mathrm{pa}-$ tients $(50 \%)$ required sedation by continuous infusion of midazolam before death. In patients with severe respiratory 


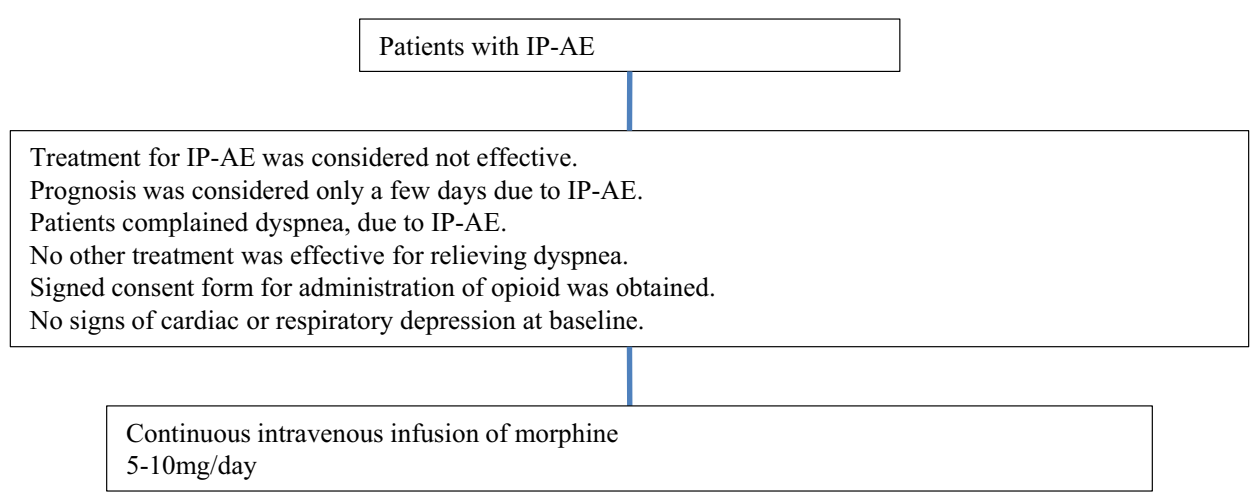

Figure 2. Morphine infusion protocol.

Table 3. Efficacy of Morphine.

\begin{tabular}{llll}
\hline & $\begin{array}{l}\text { All patients } \\
\text { receiving } \\
\text { morphine } \\
(\mathrm{n}=22)\end{array}$ & $\begin{array}{l}\text { Patients receiving } \\
\text { morphine whose } \\
\text { oxygen saturation } \\
\geq 85 \%(\mathrm{n}=12)\end{array}$ & $\begin{array}{l}\text { Patients receiving } \\
\text { morphine whose oxygen } \\
\text { saturation }<85 \% \\
(\mathrm{n}=10)\end{array}$ \\
\hline Efficacy & & & \\
Good & 9 & 6 & 3 \\
Moderate & 8 & 5 & 3 \\
Poor & 4 & 1 & 3 \\
Unknown & 1 & 0 & 1 \\
Efficacy rate $(\%)$ & 77 & 92 & 60 \\
\hline Data are numbers unless otherwise indicated. & &
\end{tabular}

failure and oxygen saturation $<85 \%$ before starting morphine, the efficacy rate of morphine was $60 \%$. Conversely, in patients with oxygen saturation $\geq 85 \%$ before starting morphine, the efficacy rate of morphine was $92 \%$ (Table 3 ).

Safety was also evaluated. Among 17 patients who were alive at 24 hours after starting morphine, six patients were excluded from the safety evaluation due to the lack of data regarding their vital signs. Therefore, we analyzed 11 patients whose vital signs were obtained before, and 12 hours after, they starting receiving morphine (Table 4). There was a significant change in the respiratory rates; however, no patients had fewer than eight respirations per minute. There was no significant change in the systolic blood pressure. The major adverse event was sedation (19 of 22 patients; among those, two fell asleep within 24 hours).

\section{Discussion}

This study showed that continuous intravenous morphine infusion was effective in $77 \%$ of the patients studied in relieving severe dyspnea associated with the terminal stage of AE-IP, and continuous intravenous morphine infusion lowered the respiratory rates of the patients substantially without respiratory depression. Generally, respiratory physicians are reluctant to use opioids in patients with respiratory failure and tend to use them after dyspnea has become very severe; however, the result of this study suggests that earlier intervention with morphine for dyspnea might be effective and safe for these patients.

Opioids, including morphine and dihydrocodeine, are
Table 4. Median of Physiological Variables before Treatment with Morphine (Baseline) and after 12 Hours ( $n=11)$.

\begin{tabular}{lrrr}
\hline & Baseline & $12 \mathrm{~h}$ & $\mathrm{p}$ value \\
\hline Respiratory rate per minute & $25(20-39)$ & $17(14-24)$ & 0.02 \\
Systolic blood pressure in mmHg & $130(104-148)$ & $122(100-129)$ & 1.0 \\
Oxygen saturation in percent & $91(90-94)$ & $91(88-94)$ & 0.2 \\
\hline Data are median values (interquartile range). & &
\end{tabular}

known to be effective to relieve dyspnea by one or more mechanisms, including decreasing the respiratory drive and altering the central perception and activity of peripheral opioid receptors located in the lung; clinically, opioids have been shown to decrease anxiety $(12,13)$. To the best of our knowledge, no sufficient data are available on the use of other opioids, such as oxycodone and fentanyl. A recent Cochrane systematic review found no evidence that benzodiazepines relieved dyspnea in patients with COPD. Thus, morphine has been widely used to relieve dyspnea as a firstline drug, primarily for patients with cancer. Although palliative care medicine has predominately focused on the management of patients with cancer at the end of life, recent guidelines demand that palliative care embrace all patients with life-limiting disease, irrespective of the diagnosis (14-16).

Evidence increasingly supports the benefits of opioids in treating dyspnea in patients with non-malignant lung diseases such as COPD. Although IP has a high mortality and symptom burden, data on the efficacy and safety for IP patients are lacking. To the best of our knowledge, this is the first report to show that morphine is effective in an IP patient population in relieving dyspnea and substantially lowering the respiratory rates of the patients without respiratory depression. The major adverse event was sedation, although we consider that sedation has some effect in relieving dyspnea. All deaths were considered to be primarily the result of respiratory failure due to IP. Whether morphine therapy shortened the survival is uncertain, given that we could not conduct a prospective study to compare the prognosis within a placebo study. However, given that all patients who could communicate had severe and refractory dyspnea and that less risky treatments are not available in the situation, we believe that it is ethically justified to use opioids carefully, 
under close observation, to relieve suffering in these patients.

Our study was relatively small, because IP is a rare disease, the prevalence of IPF is reported to be 10.0 per 100,000 population (17), and death from acute exacerbation is not a common event in a single center. Our study was a non-controlled, non-blinded, retrospective study in design for two reasons. First, ethically, we could not conduct a prospective, controlled, blinded study for the patients who died within a few days and who knew they were dying. Second, we could not find sufficient evidence to conduct a prospective study. The numerical rating scale, which is commonly used to measure dyspnea severity, was unavailable because the symptoms was too severe and patients could often barely speak; therefore, a potential limitation associated with this study could be the methodology. Nevertheless, there is an acknowledged paucity of published data, and the reason is that a prospective, controlled, blinded study is hard to conduct ethically, and quantitative measures are not reliable and nearly impossible in this severe setting. We believe that the observations reported in this paper contribute to a clearer understanding of the use of opioids in end-stage AE-IP, and larger, prospective studies are required for an expanded evidence base on which to base clinical decision-making for IP patients with dyspnea, including patients with not only $\mathrm{AE}$ or end-stage patients, but also chronic and subacute exacerbation and earlier stage patients in the future.

We conclude that continuous morphine infusion is an effective and safe therapy for severe dyspnea in patients with terminal stage AE-IP.

The authors state that they have no Conflict of Interest (COI).

\section{Financial Support}

This study was supported by a Grant-in-aid from the Japanese Ministry of Health, Labour and Welfare.

\section{References}

1. Churg A, Wright JL, Tazelaar HD. Acute exacerbations of fibrotic interstitial lung disease. Histopathology 58: 525-530, 2011.

2. Collard HR, Moore BB, Flaherty KR, et al. Acute exacerbations of idiopathic pulmonary fibrosis. Am J Respir Crit Care Med 176: 636-643, 2007.

3. Bruera E, MacEachern T, Ripamonti C, Hanson J. Subcutaneous morphine for dyspnea in cancer patients. Ann Intern Med 119: 906-907, 1993.
4. Abernethy AP, Currow DC, Frith P, Fazekas BS, McHugh A, Bui C. Randomised, double blind, placebo controlled crossover trial of sustained release morphine for the management of refractory dyspnoea. BMJ 327: 523-528, 2003.

5. Currow DC, McDonald C, Oaten S, et al. Once-daily opioids for chronic dyspnea: a dose increment and pharmacovigilance study. J Pain Symptom Manage 42: 388-399, 2011.

6. Rocker G, Horton R, Currow D, Goodridge D, Young J, Booth S. Palliation of dyspnoea in advanced COPD: revisiting a role for opioids. Thorax 64: 910-915, 2009.

7. Allen S, Raut S, Woollard J, Vassallo M. Low dose diamorphine reduces breathlessness without causing a fall in oxygen saturation in elderly patients with end-stage idiopathic pulmonary fibrosis. Palliat Med 19: 128-130, 2005.

8. Japanese Respiratory Society's Committee formulating diagnosis and treatment guideline for diffuse lung diseases. In: [Clinical Diagnostic and Treatment Guidance for Idiopathic Interstitial Pneumonias]. 2nd ed. Nankodo, Tokyo, 2011: 67-73 (in Japanese).

9. Song JW, Hong SB, Lim CM, Koh Y, Kim DS. Acute exacerbation of idiopathic pulmonary fibrosis: incidence, risk factors and outcome. Eur Respir J 37: 356-363, 2011.

10. Matsuo N, Morita T. Efficacy, safety, and cost effectiveness of intravenous midazolam and flunitrazepam for primary insomnia in terminally ill patients with cancer: a retrospective multicenter audit study. J Palliat Med 10: 1054-1062, 2007.

11. Lanken PN, Terry PB, DeLisser HM, et al. An official American Thoracic Society clinical policy statement: palliative care for patients with respiratory diseases and critical illnesses. Am J Respir Crit Care Med 177: 912-927, 2008.

12. Jennings AL, Davies AN, Higgins JPT, Gibbs JSR, Broadley KE. A systematic review of the use of opioids in the management of dyspnoea. Thorax 57: 939-944, 2002.

13. Woodcock AA, Gross ER, Gellert A, Shah S, Johnson M, Geddes DM. Effects of dihydrocodeine, alcohol, and caffeine on breathlessness and exercise tolerance in patients with chronic obstructive lung disease and normal blood gases. N Engl J Med 305: 16111616, 1981.

14. Boland J, Martin J, Wells AU, Ross JR. Palliative care for people with non-malignant lung disease: summary of current evidence and future direction. Palliat Med 27: 811-816, 2013.

15. Department of Health. End of life care strategy: promoting high quality care for all adults at the end of life. London: 2008 [Internet]. [cited May 1, 2015]. Available from: www.gov.uk/governmen t/uploads/system/uploads/attachment_data/file/136431/End_of_life_ strategy.pdf

16. Higginson IJ, Bausewein C, Reilly CC, et al. An integrated palliative and respiratory care service for patients with advanced disease and refractory breathlessness: a randomised controlled trial. Lancet Respir Med 2: 979-987, 2012.

17. Natsuizaka M, Chiba H, Kuronuma K, et al. Epidemiologic survey of Japanese patients with idiopathic pulmonary fibrosis and investigation of ethnic differences. Am J Respir Crit Care Med 190: 773-779, 2014.

(C) 2016 The Japanese Society of Internal Medicine

http://www.naika.or.jp/imonline/index.html 